\section{Wavelength generalization and discrimination in the pigeon*}

\author{
PATRICIA M. BLOUGH \\ Brown University, Providence, Rhode Island 02912
}

This three-part study describes wavelength generalization gradients around a series of training wavelengths ranging from 480 to $645 \mathrm{~nm}$. Luminance was controlled for the pigeon's spectral sensitivity. The response measure was probability of keypecking during a 2-sec stimulus presentation. Both an extinction procedure, where stimulus wavelengths occurred in $15-\mathrm{nm}$ steps, and a maintained discrimination procedure, where step size was 2 to $4 \mathrm{~nm}$, were used to obtain gradients. During a portion of the maintained discrimination procedure, new luminances were introduced, so that the effect of luminance level on gradient slope could be examined. Comparison of the resulting functions across training wavelengths revealed: (1) consistent differences in gradient slope in different spectral regions and (2) an increase in slope with luminance increase. The findings are related to recent electroretinographic wavelength contrast data and to psychophysical measures of wavelength discriminability.

The way in which discriminability relates to stimulus generalization, although much discussed in the past, has remained unclear. Some principal experiments in this field have dealt with the wavelength dimension in pigeons (Guttman \& Kalish, 1956) and in humans (Kalish, 1958). These studies examined generalization gradients in various areas of the spectrum and compared the findings with appropriate wavelength discrimination functions. The human data revealed an apparent relation between two measures; that is, the generalization gradients were steeper in those spectral regions where best wavelength discrimination occurred. On the other hand, the pigeon gradients did not appear to be steeper in the "more discriminable" portions of the spectrum. More recently, Marsh (1967) has suggested that, for the pigeon, the generalization/discriminability relation is affected by the number of stimuli presented during the testing procedure.

With the better controls and techniques that are now available, it seems feasible to have a second look at this problem. An important consideration, for example, is the control of luminance, which was confounded with wavelength in the Guttman and Kalish study. Several studies of the pigeon photopic spectral sensitivity function (D. Blough, 1955; Graf, 1969; P. Blough, Riggs, \& Schafer, 1972) have yielded such similar results that we can now, with confidence, adjust lights of varying

*This research was supported in part by USPHS Grant MH 02456. The author is indebted to Donald Blough for his suggestions and comments and to Lynn Aikin, Patrick Mulvanny, Virginia Vaughan, and Dean $Y$ ager for their assistance in various phases of these experiments. wavelength so that they are equal in luminance for the piegon eye. There are also available for pigeons two new wavelength discrimination functions, one based on a behavioral study (A. Wright, 1972) and one derived from electroretinographic (ERG) data of Riggs, P. Blough, and Schafer (1972). Both of these curves are quite different from the older Hamilton and Coleman (1933) data, referred to by Guttman and Kalish. Comparing the new functions with wavelength generalization gradients obtained under constant luminance by $D$. Blough (1961), we find at least a hint of correlation between slope and discriminability.

In examining the generalization/ discrimination problem, however, it is important to bear in mind that different techniques yield different gradient shapes. It is well known, for example, that the slope of the generalization gradient becomes steeper when the amount of discrimination training is increased (Jenkins \& Harrison, 1960). A method described by $D$. Blough (1969) allows us to obtain steep gradients over a restricted range of stimuli by maintaining the discrimination with occasional reinforcements during the testing period. This type of gradient permits comparison of response probabilities over a relatively narrow range of stimuli. If, on the other hand, we wish to compare responding over a broad stimulus range, the more traditional method of obtaining data in the course of extinction is appropriate.

The present study is concerned with wavelength generalization in various parts of the spectrum under controlled luminance conditions. Using the extinction procedure, we have obtained a set of broad gradients suitable for comparison with the Riggs, Blough, and Schafer (1972) study, which examined responses to relatively large wavelength differences. The maintained discrimination method has yielded sets of narrower functions, which may be related to the wavelength discrimination function of Wright (1972). Finally, we have examined the effect of luminance level on the shape of gradients obtained in a maintained discrimination.

\section{APPARATUS}

The basic components of the apparatus were a set of three similarly equipped Grason-Stadler pigeon boxes, an optical system controlling wavelength and the illumination of the response key, and computer-controlled relay circuitry. Each pigeon chamber contained a single plastic response key, which was partially blackened so that only its center was illuminated by the stimulus light. This aperture was circular and had a diameter of $2 \mathrm{~mm}$. The magazine light, a $6-\mathrm{W} 125-\mathrm{V}$ bulb, was painted black in order to reduce illumination and thus the effects of chromatic adaptation-during reinforcement. No other sources of light were present in the box during the experimental sessions. A fan provided ventilation and a loudspeaker, a source of continuous white noise.

A Bausch and Lomb 250-mm grating monochromater provided the monochromatic lights that served as stimuli. Entrance and exit slits were set at $2 \mathrm{~mm}$; nominal half bandwidth was $6.6 \mathrm{~nm}$. The light source was a Sylvania DNF tungsten-halogen 150-W bulb operated at $6.5 \mathrm{~A}$. Light from the monochromater passed through a neutral density wedge and entered three 3-mm fiber optics light guides, one of which led to the back of each response key. A single shutter controlled stimulus presentation for all three boxes.

The principal calibrating instrument was a Photovolt photomultiplier aimed directly at the pigeon's side of the response key. Readings at $10-\mathrm{nm}$ steps from 430 to $650 \mathrm{~nm}$ were corrected for the photomultiplier's spectral sensitivity to provide relative energy data at the key. Photomultiplier readings also yielded curves describing the transmission characteristics of the neutral density wedge. Using the spectral sensitivity data of $D$. Blough (1955), it was then possible to calculate wedge settings such that all stimulus wavelengths would have equal luminance for the pigeon eye. (The Blough data were used here because they were taken at luminance levels most closely resembling the present values.) Measurements with a MacBeth illuminometer provided data on the 


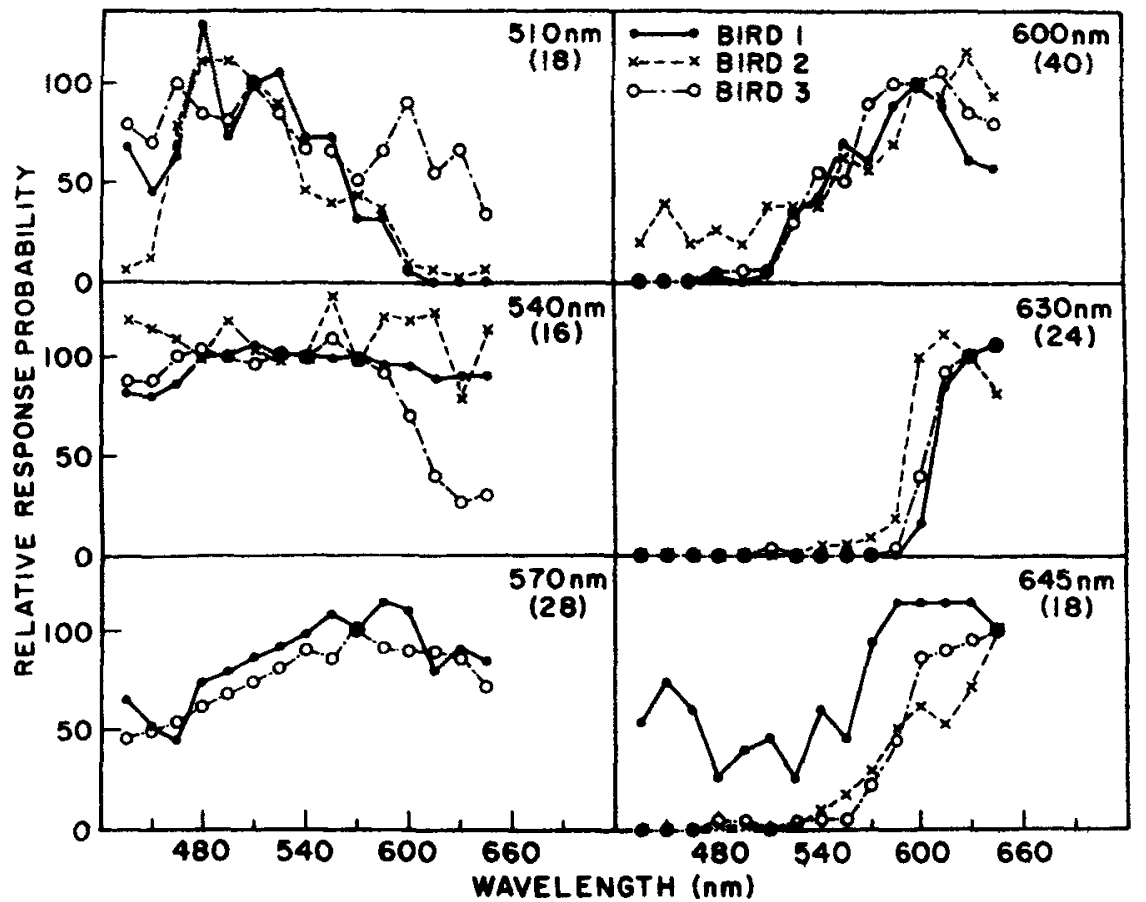

Fig. 1. Individual generalization gradients for the extinction procedure. Included are only those Ss that emitted a total of 15 or more responses to the training stimulus during the combined extinction sessions. No $S$ gave more than 60 responses to the positive stimulus. Numbers in parentheses indicate total training sessions for that group.

absolute luminance level. Since the target was too small to measure directly, it was necessary to adjust the luminance of a larger adjacent field until it appeared to match the stimulus and then to measure this larger field with the MacBeth. The luminance value measured at $580 \mathrm{~nm}$ for the human eye was $.058 \mathrm{~mL}$ in Box 1 , $.032 \mathrm{~mL}$ in Box 2 , and $.021 \mathrm{~mL}$ in Box 3.

A LINC computer (Clark \& Molnar, 1964) controlled wavelength and energy adjustments by operating the motor-driven wedge and monochromater. The computer also controlled the shutter and food magazine, performed the various timing operations, and sensed responses. Additional programs analyzed and printed out the data.

\section{SUBJECTS}

The Ss were 22 pigeons of mixed breed and sex. A separate group of birds was used at each training wavelength. In most cases, a group consisted of three pigeons, but in two instances there were only two in a group. (Figures and legends give details.) Birds trained at a given wavelength usually served as Ss for both the extinction procedure and the corresponding maintained gradient procedure. All birds were maintained at approximately $75 \%$ of their ad lib weights.

\section{GRADIENTS OBTAINED IN EXTINCTION Procedure}

An initial training procedure shaped the birds to peck at the training wavelength in a dark box with the dim light illuminating the magazine during reinforcement. After a preliminary session or two of magazine training with a bright light, the magazine light was dimmed and key training begun. This was accomplished by the conventional shaping procedures in some cases and, in others, by an "autoshaping" method (Brown \& Jenkins, 1968). The autoshape program was made up of a series of 8 -sec presentations of the keylight, each followed by a 6-sec reinforcement period. These presentations occurred on a variable-interval schedule independent of the pigeon's behavior. During this autoshaping procedure, each keypeck also operated the food magazine. In all cases, key training occurred in the presence of the training wavelength at the luminance given above.

When key training was complete, the birds began to work on a schedule intended to maintain the response with a relatively low probability of reinforcement. Two-second presentations of the stimulus light alternated with an intertrial interval that lasted $5 \mathrm{sec}$ if the response was not reinforced and $12 \mathrm{sec}$ when reinforcement occurred. In the latter case, the intertrial interval began when the magazine had closed. The purpose of this relatively long postreinforcement interval was to help minimize effects of chromatic adaptation from the magazine light. Since a single optical system served all three pigeon chambers, stimulus presentations were synchronized for all birds in a group. One or more pecks by any bird during the intertrial interval delayed stimulus onset by $1 \mathrm{sec}$, and every peck during the delay period itself reset the delay timer so that stimulus onset followed such pecks by 1 sec.

Reinforcement probability gradually decreased across sessions. During the first 3 or 4 days after key training, it had a value of 0.5 ; it then decreased through values of 0.25 , 0.125 , and 0.062 to a final value of 0.031 . These values denote the probability that a single peck during a given stimulus presentation would operate the magazine. The occurrence of reinforcement was determined independently for each trial by a random process, except that a maximum was set on the number of successive unreinforced trials. This maximum increased from 4 at first to a final value of 48 or 64. Magazine time varied among birds and ranged from 4 to 8 sec. For each bird, the duration was the value that allowed the bird to maintain its $75 \%$ weight without supplementary food.

The number of trials in a session increased as reinforcement probability decreased. While the number of reinforced trials had a maximum of about 65 , the number of unreinforced trials started at 60 and increased to 900 when the final reinforcement condition was reached. Data for these sessions were evaluated simply in terms of the ratio of responses to trials. (The first 30 trials were not included, however, because of possible warm-up effects.). Only the first response in each trial was recorded. Binds were run on each reinforcement probability until all birds responded on most trials.

Training wavelength values ranged from 510 to $630 \mathrm{~nm}$ in $30-\mathrm{nm}$ steps, and there was, in addition, a group run at $645 \mathrm{~nm}$. The total number of training sessions varied among groups from 16 to 40 sessions, since the different groups met the training criterion described above at different rates (see Fig. 1 for details).

Testing occurred in extinction. Fifteen test values occurred in 15-nm steps ranging from 435 to $645 \mathrm{~nm}$; thus, the training wavelength was always a part of the test series. During the test session, these 15 wavelengths were presented in a randomized blocks 
design, with a single block consisting of the 15 test values. The energy of each test wavelength was adjusted by the neutral density wedge so that all stimuli were equal in luminance for the pigeon eye. There was a total of 900 trials in the first extinction session. A second session followed, usually with one or more training sessions intervening. Each test wavelength was presented equally often during the testing procedure.

\section{Results and Discussion}

Individual generalization gradients for the extinction procedure appear in Fig. 1. These broad functions show responding over a considerably wider range than much of the other published data (e.g., Guttman \& Kalish, 1956; D. Blough, 1961), and some of the individual functions show a good deal of variability. The relatively low luminance of the stimuli, the wide range of the test stimulus set, the use of a "go/no-go" criterion, and a rather small number of responses may account for these characteristics. Though unsuitable for clarifying the fine details of wavelength control, the data are useful for comparing response probability over a broad stimulus range.

The individual data reveal clear-cut differences in gradient shape and steepness. The averaged data, appearing as solid lines in Fig. 2, summarize these effects. The gradients around 540 and $570 \mathrm{~nm}$ are relatively flat, while response probability falls off especially rapidly to the left of the 630- and the 645-nm functions. The number of training sessions does not appear to contribute to these differences.

One way to evaluate these findings is in terms of color-naming data. Wright and Cumming (1971) have described a set of "pigeon hues," and Wright (1972) has suggested that discriminability is best at the boundaries between these hues. The present design might be expected to yield relatively flat gradients when the training stimulus is in the center of a hue region. The gradients would come to a sharp peak around a training stimulus at the hue boundaries; and, when the training wavelength is near, but not at, a hue boundary, we might expect a sharp fall in the gradient in the direction of that boundary. Some of the present data appear to support the $600-\mathrm{nm}$ boundary postulated by Wright and Cumming. The 600- $\mathrm{nm}$ gradient comes to a sharp peak at that wavelength and falls off symmetrically in both directions; furthermore, the 630- and 645-nm gradients fall quite steeply in the $600-\mathrm{nm}$ region. We also note that the slope of the $540-\mathrm{nm}$ gradient becomes steep in that area;

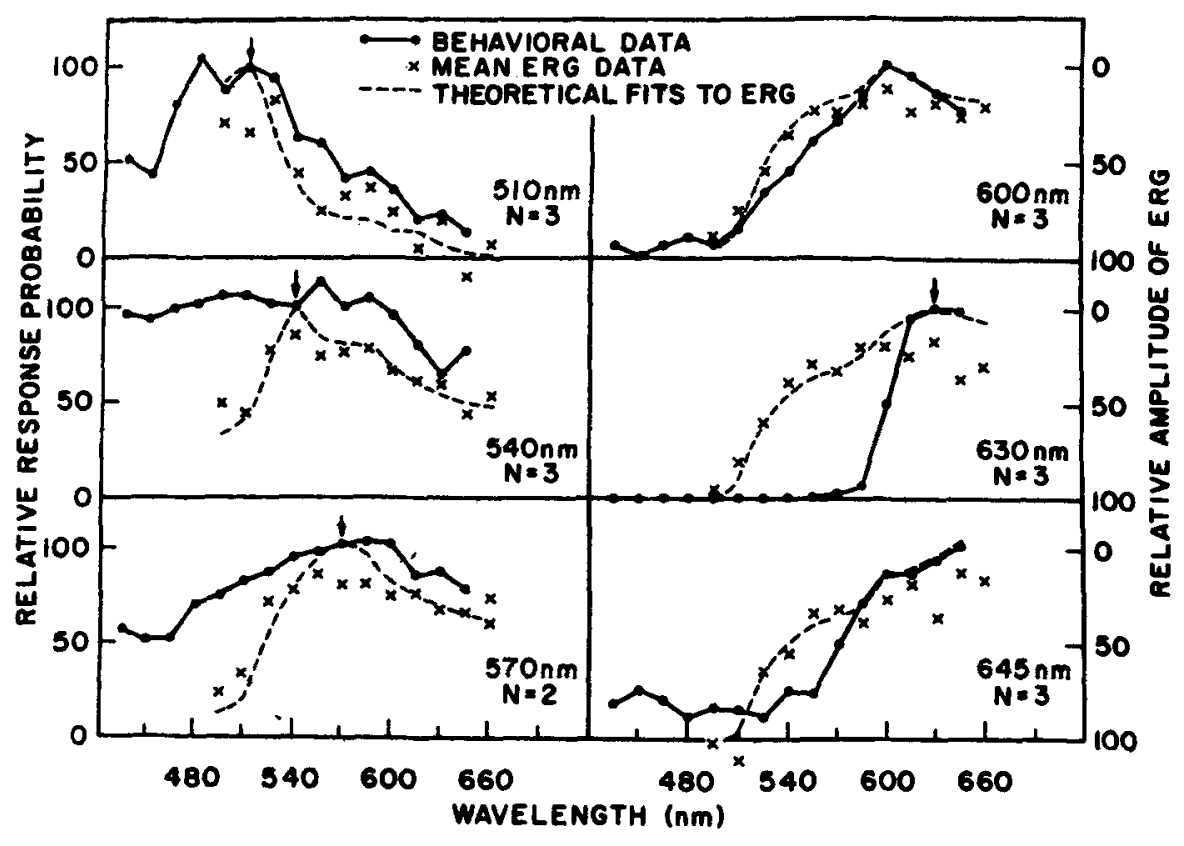

Fig. 2. Solid lines are the extinction gradients averaged across Ss. The ERG data and functions are based on a procedure described in the text and, more fully, in Riggs, Blough, and Schafer (1972).

the individual data for this wavelength show this tendency to be very marked for one bird, while the others have flat gradients throughout, suggesting little or no stimulus control in their cases. The $570-\mathrm{nm}$ data do not fit the notion of a $600-\mathrm{nm}$ boundary, however.

Wright and Cumming postulate a second hue boundary at $540 \mathrm{~nm}$, but the present results do not fit as well with that notion. The 540-nm gradient is flat rather than having the pointed shape we might expect if such a boundary were present, and the 570-nm gradient fails to fall off steeply in that direction either. The shape of the 510-nm gradient suggests the presence of this wavelength in a hue region set off from both extremes of the spectrum. It is hard to explain, however, why these birds failed to generalize in the direction of the longer wavelengths, while the 540-nm birds generalized so readily toward the shorter values. Perhaps individual differences in overall gradient steepness are confounding these findings. It was necessary to use different birds at each training wavelength in order to avoid serial learning effects. Yet this procedure loses the advantages of using $\mathrm{Ss}$ as their own controls.

The ERG data of Riggs, Blough, and Schafer (1972) are plotted with the generalization functions in Fig. 2. These curves describe the amplitude of the electroretinographic response when the eye is stimulated by alternating pairs of monochromatic lights equal to each other in luminance. For example, the graph labeled $510 \mathrm{~nm}$ shows the change in response as the eye is stimulated by a 510-nm light alternating with a series of other wavelengths, while the graph labeled $540 \mathrm{~nm}$ shows the effect of alternating a 540-nm light with other lights across the spectrum. Note that these data are plotted upside down for convenience, since ERG amplitude decreases from the bottom to the top of each graph. Thus, the functions show that the response increases as the wavelength pairs become physically more separated. We have shown in the graphs both the averaged ERG data points and the smooth fits reached by applying a trichromatic theory of wavelength discrimination.

In comparing the ERG and the behavioral functions in Fig. 2, we note that, at 510,540, and $570 \mathrm{~nm}$, the ERG curves tend to have steeper slopes, while the generalization gradients are steeper at the three longer wavelengths. More specifically, in the $525-\mathrm{nm}$ region, where wavelength differences appeared to have a relatively large effect on the ERG, the pigeon tended to generalize readily from one wavelength step to another. On the other hand, the birds' failure to generalize was most pronounced across the region extending below and around $600 \mathrm{~nm}$, where the retina was less affected by wavelength differences.

Because of the many differences between the ERG and the behavioral procedures, this comparison must be interpreted cautiously. The ERG study 


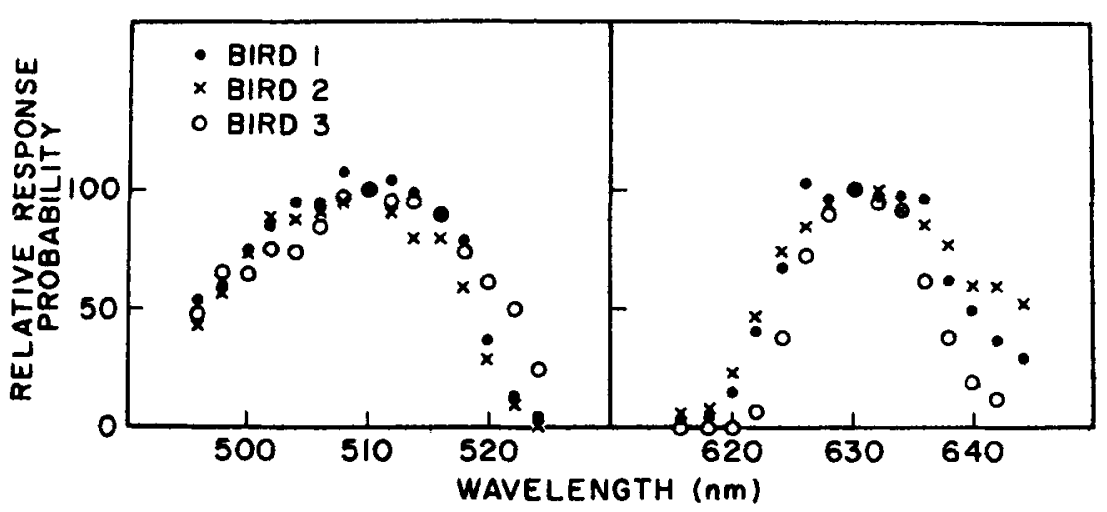

Fig. 3. Samples of individual gradients around 510 and $630 \mathrm{~nm}$ obtained with the maintained procedure. Different groups of birds were used at the different training wavelengths.

used substantially higher luminance levels, and the response was to rapidly alternating wavelength pairs. In the behavioral work, each response was to a single wavelength at a rather low luminance. Most important, of course, is the fact that the ERG response comes from activity in the distal portion of the retina, while the behavioral response involves the entire visual system. Since both studies concern responses to wavelength differences, it is tempting to speculate on the role of visual processing in producing the differences shown in Fig. 2.

The broad gradients shown in this portion of the study leave unanswered more detailed questions about gradient shapes in various spectral regions. The next experiment examines sets of relatively narrow gradients.

\section{GRADIENTS OBTAINED DURING \\ A MAINTAINED DISCRIMINATION Procedure}

Since most of the birds used in this portion of the study were the same as those used in the extinction procedure, they had already had extensive experience with the positive stimulus when this procedure began. The discrete trial method was also continued in this phase of the experiment. The major changes were a response requirement of three pecks instead of one and the presentation of 15 stimulus wavelengths throughout. The randomized blocks design for presentation of the various wavelength values was that used during the extinction testing described above. These values included presentations of the training stimulus, which fell in the middle of the series of stimulus wavelengths, spaced at 2- or 4-nm steps. Again, the luminance of the various stimuli was constant for the pigeon eye.

Reinforced presentations of the training wavelength were programmed independently of the randomized presentations. These reinforced trials occurred with probabilities ranging from 0.5 to 0.031 and with maximum consecutive unreinforced trial limitations ranging from 4 to 64 . The data include responses to unreinforced presentations of the training stimulus, but not to the reinforced

Except for the addition of a $480-\mathrm{nm}$ group and the elimination of the 645-nm group, the training wavelengths were those used previously. The goal of this experiment was to compare gradients taken at comparable training levels across groups. (A comparison of "steady-state" gradients did not seem feasible, since many weeks of training are required to reach a stable level and because such gradients may become so sharp that differences among them may be obscured.) Thus, training procedures were held approximately constant for all groups. Initial sessions used a reinforcement probability of 0.5 and a set of stimulus wavelengths spaced $2 \mathrm{~nm}$ apart. During these sessions, the ratio requirement was When the birds were responding regularly on the new procedure, a series of sessions at gradually decreasing reinforcement probabilities began. The first of these was a session at a probability of 0.25 ; then followed two sessions at a probability of 0.125 , two at a probability of 0.062 , and the final condition at a probability of 0.031 . These sessions continued until the birds reached a criterion level of stability where the ratio of responses at the training wavelength to total responses did not change by a factor of $2 \%$ or more over the course of two successive sessions. Two more sessions followed in which data were saved and analyzed. The next group of sessions used the same reinforcement probability of 0.031 , but the stimulus presentations. increased from one to three pecks. wavelengths were spaced at $4-\mathbf{n m}$ steps. Again, the birds were run until they achieved criterion performance and then through two additional sessions for data analysis. At this point, there was in most cases a series of two to four sessions to test for luminance effects (see next section). Now the spacing of stimulus wavelength returned to $2-\mathrm{nm}$ steps, and two sessions of data were again collected for analysis. For the 570and 600-nm birds, luminance testing occurred at this point rather than during the earlier period described above.

\section{Results and Discussion}

Figure 3 illustrates some individual data from this portion of the experiment. Relative to the extinction data, there is considerably less variability among individual Ss. Furthermore, the functions for each bird show fewer reversals; that is, there is a more consistent relationship between wavelength and response probability. Since it was possible to maintain responding by the randomly introduced reinforced trials in this procedure, the peak data points here represent many more responses than was the case in the extinction procedure. In this experiment, for example, total responses to unreinforced presentations of the positive stimulus were in the neighborhood of $80-120$. It is likely that this increase in sample size accounts for much of the reduced variability.

Figure 4 shows, by solid and dashed lines, sets of gradients taken during the second and third periods of data analysis, respectively. (The data from the first analysis period are not shown here, since many of the gradients were so flat that comparisons were difficult.) The dashed gradients are clearly steeper than those represented by the solid line, and we may account for this difference at least partly by the fact that the former data were taknn at a somewhat later stage in training. It is likely that further training would have sharpened up these functions even more. The differences between the two sets of gradients may also relate to differences in wavelength step size.

Despite slope differences, there are some striking similarities between the earlier and the later gradients. Notable among these is the consistency of what appear to be training wavelength effects. The gradients at the various wavelengths differ from each other both in overall steepness and in respect to the asymmetry that characterizes the shapes of certain functions. For example, the 600-nm gradients are relatively symmetrical and clearly 
steeper than any of the others; this is true for both sets of 600-nm data. The $540-\mathrm{nm}$ gradient in both cases tends also to be symmetrical, and they are also somewhat steeper than the 480 -, $510-$ and $570-\mathrm{nm}$ gradients. Pronounced asymmetries occur in the $480-$ and $630-\mathrm{nm}$ gradients in that both sets of functions are steeper in the direction of the shorter opposite, asymmetry occurs in the 510- and 570-nm gradients, which are steeper toward the longer wavelengths.

The question of how gradient slope relates to discriminability measures now arises. Were such a relationship the case, the steepness of the 600-nm functions would suggest an area of maximum sensitivity in that region. The asymmetries of the 630- and 570-nm gradients are consistent with this view, since the steeper sides of both functions are in the direction of $600 \mathrm{~nm}$. The asymmetry of the $510-\mathrm{nm}$ gradients suggests that wavelengths longer than this value are more discriminable than the shorter wavelengths; this finding is reminiscent of D. Blough's (1961) function around $530 \mathrm{~nm}$, for that curve also was steeper in the long wavelength direction. (Blough found a reverse asymmetry in a 550-nm gradient and concluded that the pigeon was relatively sensitive to wavelength differences in the 530- to 550-nm range.) Thus, the data from this portion of the study support Wright and Cumming's suggestion that there are areas of relatively high sensitivity around their "hue boundaries" at 540 and $600 \mathrm{~nm}$. The symmetry and the wavelengths. A consistent, but

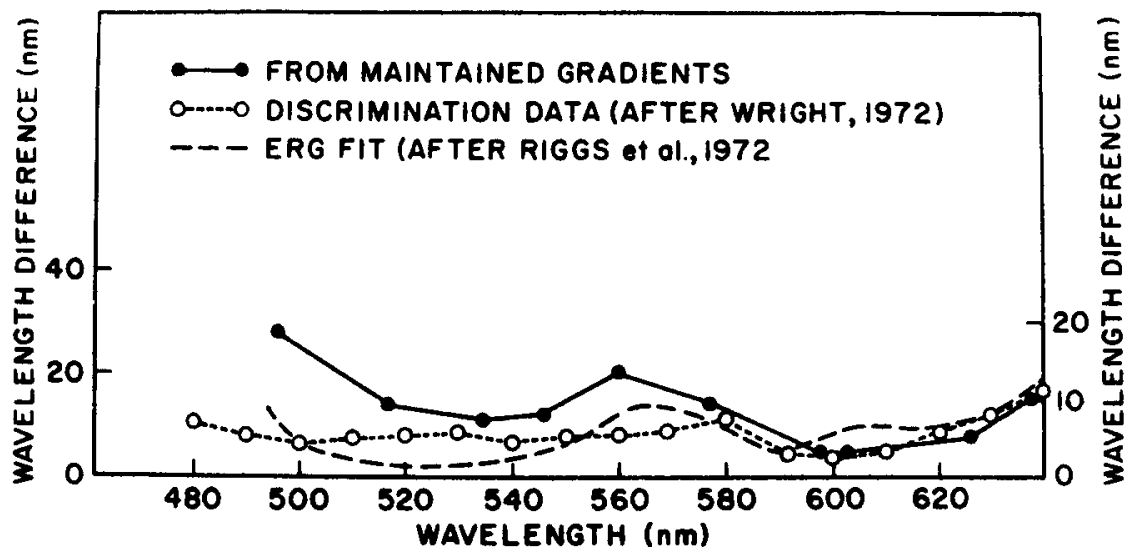

Fig. 5. Comparison of three "wavelength discrimination" functions obtained with different methods. Present data are scaled on left ordinate; Wright data are scaled on right. ERG data have arbitrary scales and are adjusted to facilitate comparison. See text for details.

relative steepness of the 540 - and 600-nm gradients are also in line with this conclusion, since these gradients would fall in the crossover portions of two "pigeon hues." The shapes of the 480-nm gradients suggest a third possible area of increased sensitivity in the short wavelength region, but the fact that neither of the gradients falls close to zero response probability indicates that sensitivity here would not be as great as in the 540- and 600-nm regions.

Not only do the present findings appear consistent with the Wright and Cumming color-naming data, but they also agree with Wright's (1972) finding that the wavelength difference limen is minimal in the 600-nm region of the spectrum. It is tempting, then, to go

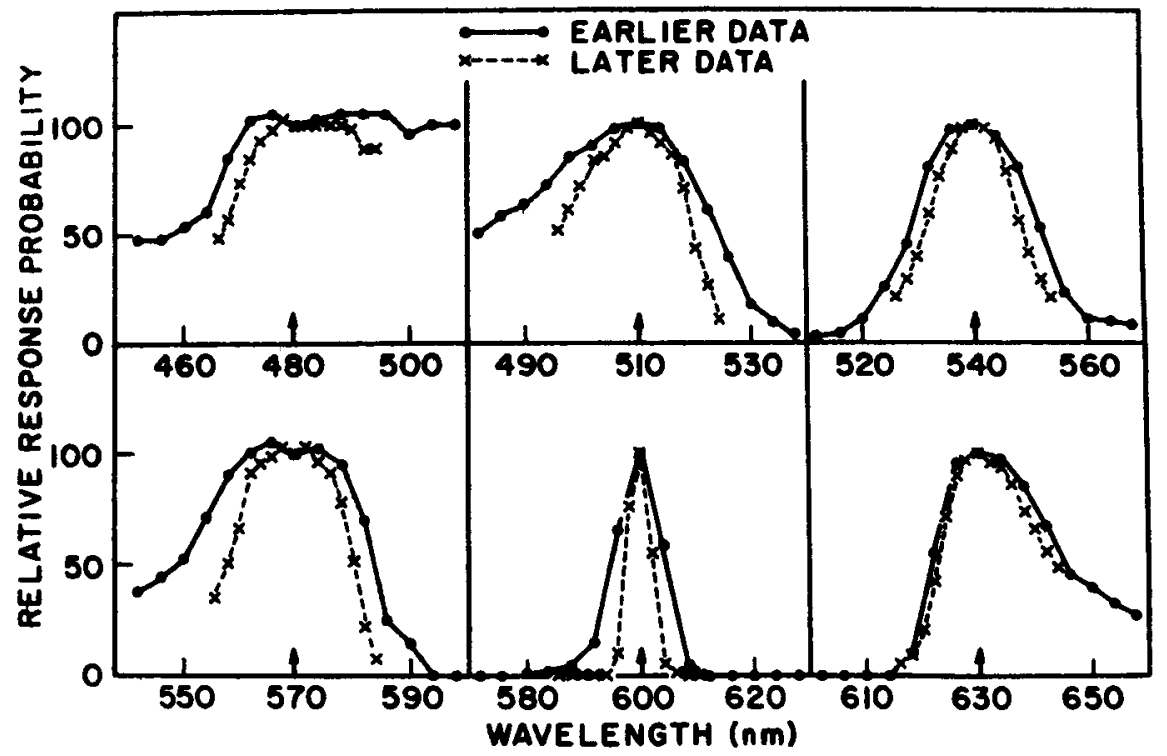

Fig. 4. Maintained gradients averaged across birds at two different stages of training. The points connected by solid lines and spaced at $4-\mathrm{nm}$ steps were taken somewhat earliex than the others. on to relate more precisely the details of gradient slopes to Wright's measures of discriminability. A drawback here is the possibility in the present study of differential training effects at the various wavelengths. Were this the case, relationships that held at one stage of training might not continue to hold at a later stage. Although training conditions were approximately the same for all groups, they were not identical. The separate functions in each section of Fig. 4 do represent somewhat different parts of the training period, however, and it is encouraging that they agree as much as they do. A Spearman rank order correlation coefficient between the two sets of data was .953. (This statistic was based on the ordinate values crossed by the various functions at a distance of $10 \mathrm{~nm}$ from the training wavelength. Since it was possible to make the measurement in two directions for each of the six curves, the coefficient is based on an $N$ of 12.) The extent of the correlation suggests that consistent wavelength effects do indeed underlie the various gradient shapes.

Figure 5 compares three "wavelength discrimination" functions. Wright's data are based on a threshold technique in which wavelength difference limens were determined at $10-\mathrm{nm}$ intervals across a large portion of the spectrum. The Riggs, Blough, and Schafer function, as described earlier, was derived from electroretinographic data. The present findings were converted into points on a "wavelength discrimination" function by adopting a criterion of response probability $(50 \%)$ and finding the distance from the training wavelength at which the generalization gradient fell to this level. (These determinations were based on the solid lines shown in Fig. 4.) The points, of 


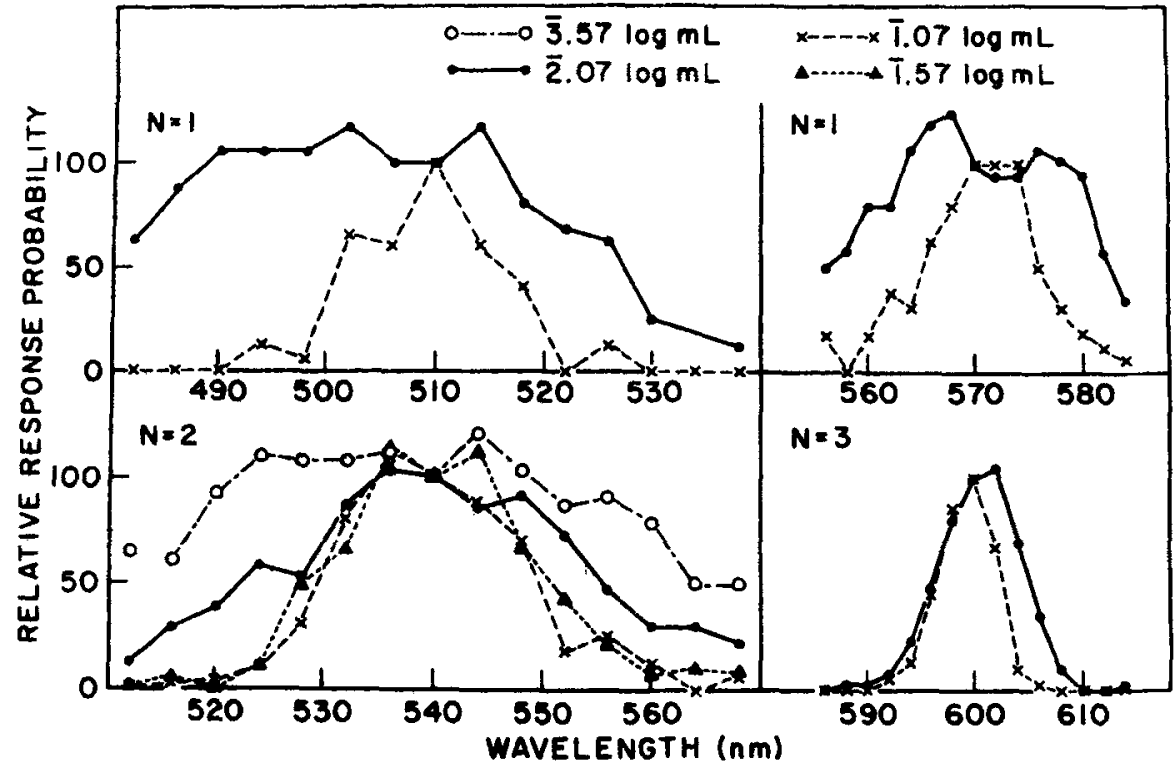

Fig. 6. Gradients obtained at different luminance levels at each of four wavelengths. Number of Ss averaged at each wavelength is indicated. The set of functions at each wavelength was obtained at the same stage of training. However, training is not comparable between wavelengths; thus, comparisons of shape to training wavelength are inappropriate here. Luminance figures are averaged values for the three pigeon chambers.

course, do not represent a "threshold"; it is the relationship among them that should be noted. This relationship, of course, would depend somewhat on the choice of criterion response level. Hopefully, different criteria would not alter the function too much. In fact, the present curve is very similar to one derived from the dashed lines of Fig. 4 by plotting the response probabilities yielded at points $10 \mathrm{~nm}$ to either side of the training wavelengths.

Comparisons among the three functions in Fig. 5 indicate, for one thing, a striking agreement between the two sets of behavioral data to the effect that maximum sensitivity occurs around $600 \mathrm{~nm}$. With regard to the region extending from 495 to about $580 \mathrm{~nm}$, however, the present data seem to agree more with the ERG findings, although the latter do not show so pronounced a dip around $600 \mathrm{~nm}$. It is hard to explain the relative flatness of the Wright function in the 500- to 580- $\mathrm{nm}$ region, since the present evidence, the ERG data, and the earlier D. Blough data (1961) all combine to suggest sensitivity changes through that spectral area. A possible factor is the use by Wright of very small wavelength differences. Slight individual differences in the area of maximum sensitivity might have tended to cancel each other out in the averaged data shown here. Indeed, Wright's individual functions suggest that this is the case, but even here the inflections are small.
The last portion of the study examines the role of discriminability on gradient shape by considering luminance effects.

\section{LUMINANCE EFFECTS Procedure}

Near the end of the procedure described in the preceding section, a series of two to four special test sessions were run. During these sessions, all wavelength values occurred in the usual fashion, but their luminances varied. In a given session, three luminance values occurred: the training value (approximately $\mathbf{2 . 5 7} \mathrm{log}$ $\mathrm{mL}$ ) and two test values, which were displaced one above and one below the training value either by 0.5 log units, $0.6 \mathrm{log}$ units, or $1.0 \mathrm{log}$ units. The test luminance values were not always the same at the different training wavelengths; details are in Fig. 6. In a typical test session, each of the 15 test wavelength values was presented 60 times; thus, since there were three luminances, each wavelength-luminance combination occurred 20 times. Reinforced presentations of the training wavelength at the training luminance were random, as usual, and occurred with a probability of 0.031 . There were no reinforced presentations at any luminance but the training value.

\section{Results and Discussion}

Since no presentation of the training wavelength at the new luminance values was reinforced, the birds tended to extinguish at these new luminances. This was especially the case for the higher test luminances. The data selected for presentation here include only those Ss who gave 15 or more responses to the training wavelength at the test luminances specified.

Figure 6 shows the data selected in this fashion. Note that the results at the training luminance are omitted here, since the occurrences of reinforcement may have affected the comparability of those functions to the others. The effect of luminance on the slopes of the gradients is clear; that is, in each case, the gradients taken at the higher luminances are steeper. The nature of this effect is best illustrated by the $540-\mathrm{nm}$ data, where four luminance levels are shown. Gradient slope increases with increasing luminance level, except at the two highest values, where the curves are quite similar. This set of curves suggests that the effects of luminance may disappear at higher levels.

Human wavelength discrimination data indicate improved discriminability at higher luminance levels (e.g., Weale, 1951). The gradient changes illustrated in Fig. 6 suggest a related effect. The increase in slope could occur because of a switch from scotopic to photopic vision with increasing luminance; however, it could also be a purely photopic effect, as was apparently the case in the Weale study. Although the lowest luminance here slightly exceeded the pigeon cone threshold (D. Blough, 1955), it is possible that mesopic factors partially account for these findings. Consistent with this interpretation is the fact that the luminance effect is less pronounced at $600 \mathrm{~nm}$.

Human Ss see a shift in the apparent hue of a light of constant wavelength with a change in luminance. This effect is the Bezold-Brucke phenomenon, described, for example, by Boynton and Gordon (1965). The amount and direction of the hue shift varies with stimulus wavelength; these relationships have been accounted for by Hurvich and Jameson (1957) in terms of differential effects of luminance on opponent color systems. If it occurs in pigeons, the Bezold-Brücke phenomenon might be expected to show up in the present data as a shift in gradients taken at different luminance levels. For example, if the apparent hue of a 570-nm light shifted toward the longer wavelengths as its luminance increased, we might expect to see the gradient around the $570 \mathrm{~nm}$ shift to the right with higher luminances. A study by Aikin (1971) failed to show consistent shifts, and, unfortunately, the increased flatness of the gradients at 
the low luminances makes it difficult to examine the present curves for this effect. However, the sharp gradients yielded by the $600-\mathrm{nm}$ data suggest the presence of a slight shift toward the shorter wavelengths with increasing luminance; examination of other areas of the spectrum for this phenomenon might be feasible if the present method were combined with a measure that yielded steeper gradients.

\section{DISCUSSION}

A word about the procedures used in these studies is in order, for only the first experiment used the traditional "generalization" method. The procedure of the second experiment, where reinforced trials occurred throughout the course of testing, was, in effect, a discrimination method. The increasing sharpness of the gradients over the course of time reflects the Ss, improved discrimination performances, and we would expect that they would approach a steepness limited by the same factors that affect discriminability in threshold experiments. The gradients of Fig. 4, taken earlier in training, fall logically on a continum between generalization and discrimination, since discrimination training was incomplete. The procedure of the third experiment might be called a partially maintained discrimination, since reinforced trials occurred, but at a luminance level different from the test luminances.

The three experiments reported here suggest that each type of gradient is affected by a factor known to affect wavelength discriminability. Increasing luminance, known to improve discriminability, increased the slope of the gradients in the third study. Both the extinction procedure of the first experiment and the maintained discrimination procedure of the second revealed wavelength effects on gradient slope. To the extent that these effects are consistent with those obtained from traditional measures of discriminability, we conclude that the results of the various procedures reflect a common process.

It would seem reasonable, then, to regard the gradients shown here as further data on pigeon color vision. The solid "wavelength discrimination" function of Fig. 5 contains two minima, reminiscent of those seen in human trichromatic wavelength discrimination data. We must reason by analogy to suggest the presence of at least three color mechanisms in the pigeon, but this conclusion would be in agreement with those of Riggs, P.
Blough, and Schafer (1972) and of Wright and Cumming (1971). The consistency of the 600-nm data from this study with those of Wright (1972) indicates convincingly that, at the behavioral level, wavelength discrimination is maximal in that spectral region. The inconsistency of this finding with the ERG data of Riggs, Blough, and Schafer (Figs. 2 and 5) suggests the presence of mechanisms in the later stages of the visual system that "sharpen up" the discrimination around $600 \mathrm{~nm}$ relative to the region from 525 to $540 \mathrm{~nm}$, where the retina appears to be maximally responsive.

It is unclear as yet just what physiological mechanisms might underlie the three or more processes that account for pigeon color vision. The literature on cone pigments is conflicting (e.g., Bridges, 1962; Liebman, 1972), and the likelihood that the effects of these pigments must in some way be modified by the colored oil globules lends more confusion to the problem. Further information is also needed concerning color processing at later stages in the visual system. More data on the Bezold-Brücke effect could eventually tie in with electrophysiological evidence of opponent mechanisms (Yazulla, 1971).

This study has illustrated the usefulness of generalization methods, particularly the maintained discrimination procedure, as a technique in animal psychophysics. When training variables are carefully controlled, this method appears to provide data that reflect largely the same processes that are tapped by the more familiar discriminability measures.

\section{REFERENCES}

AIKIN, L. C An investigation of the Bezold-Brücke hue shift in the pigeon. Unpublished MA thesis, Brown University, 1971.

BLOUGH, D. S. Method for tracing dark adaptation in the pigeon. Science, 1955 , 121, 703-704.

BLOUGH, D. S. Spectral sensitivity in the pigeon. Journal of the Optical Society of America, 1957, 47, 827-833.

BLOUGH, D. S. The shape of some wavelength generalization gradients. Journal of the Experimental Analysis of Behavior, 1961, 4, 31-40.

BLOUGH, D. S. Generalization gradient shape and summation in steady-state tests. Journal of the Experimental Analysis of Behavior, 1969, 12, 91-104. BLOUGH, P. M., RIGGS, L. A., \& SCHAFER, $K$." L. Photopic spectral sensitivity determined electroretinographically for the pigeon eye. Vision Research, 1972, 12, 477-486. BOYNTON, R. M., \& GORDON, J Bezold-Brücke hue shift measured by color-naming technique. Journal of the
Optical Society of America, 1965, 55, 78-86.

BRIDGES, C. D. B. Visual pigments of the pigeon (Columba livia). Vision Research, $1962,2,125-137$.

BROWN, P. L., \& JENKINS, H. M. Auto-shaping of the pigeon's key peck. Journal of the Experimental Analysis of Behavior, 1968, 11, 1-8.

CLARK W. A., \& MOLNER, C. $E$. The LINC: A description of the Laboratory Instrument Computer. Annals of the New York Academy of Science, 1964, 115, Article 2, 653-668.

GRAF, V. S. A spectral luminosity function in the pigeon determined by flicker photometry. Psychonomic Science, 1969, 17, $282-283$.

GUTTMAN, N., \& KALISH, H. L. Discriminability and stimulus generalization. Journal of Experimental Psychology, 1956, 51, 79-88.

HAMILTON, $W$. F., \& COLEMAN, T. B. Trichromatic vision in the pigeon as illustrated by the spectral hue discrimination curve. Journal of Comparative Psychology, 1933, 15. 183-191.

HURVICH, L. M., \& JAMESON, D. An opponent-process theory of color vision. Psychological Review 1957, 64, 384-404.

JENKINS, H. M., \& HARRISON, R. H. Effect of discrimination training on auditory generalization. Journal of Experimental Psychology, 1960, 59, 246-253.

KALISH, H. I. The relationship between discriminability and generalization: $A$ re-evaluation. Journal of Experimental Psychology, 1958, 55, 637-644.

LIEBMAN, P. A. Microspectrophotometry of photoreceptors. In H. J. A. Dartnall (Ed.), Physiology of photoreceptor organs (Handbook of sensory physiology). Vol. VII/I. Berlin: Springer-Verlag, 1972.

MARSH, G. D. Inverse relation between discriminability and stimulus generalization as a function of number of test stimuli. Journal of Comparative \& Physiological Psychology, 1967, 64, 284-289.

RIGGS, L. A., BLOUGH, P. M., \& SCHAFER, I. L. Electrical responses of the pigeon eye to changes in wavelength of the stimulating light. Vision Research. $1972,12,981-991$.

WEALE, R. A. Hue-discrimination in para-central parts of the human retina measured at different luminance levels. Journal of Physiology, 1951, 113, 115-123.

WRIGHT, A. A. Psychometric and psychophysical hue discrimination functions for the pigeon. Vision Research, in press.

WRIGHT, A. A., \& CUMMING, w. W. Color-naming functions for the pigeon. Journal of the Experimental Analysis of Behavior, 1971, 15, 7-17.

YAZULLA, S. The transmission of wavelength information by the visual thalamus of pigeon (Columba livia). Unpublished doctoral dissertation, University of Delaware, 1971.

\section{NOTE}

1. The 480-nm group underwent training similar to that described for the other birds in the first phase of the study. However, they did not receive any extinction testing. The birds in the 600-nm group were not the same as the 600-nm birds described in the earlier results, but they had undergone similar training and extinction procedure.

(Received for publication May 7, 1972; revision received June 16,1972 .) 